\title{
Assignment of Electrical Properties to Power Grid Topologies
}

\author{
Eran Schweitzer \\ Arizona State University \\ eran.schweitzer@asu.edu
}

\author{
Anna Scaglione \\ Arizona State University \\ anna.scaglione@asu.edu
}

\author{
Kory Hedman \\ Arizona State University \\ kory.hedman@asu.edu
}

\begin{abstract}
Power systems are often described in terms of graphs, with various properties like load and impedance associated with the nodes and edges. These properties are coupled to the graph's topology, reflecting the great deal of engineering design in the power system. With the goal of automating the creation of usable synthetic cases, the problem of assigning these properties is considered. It is formulated as a Mixed Integer Program (MIP), which aims to minimize the angle differences between adjacent nodes in the system. Since the problem quickly balloons in size, a decomposition into smaller zones is explored, that enables scaling the problem to larger system sizes. Experiments demonstrate the efficacy and viability of the approach.
\end{abstract}

Index Terms-Assignment Problem, DC Powerflow, Synthetic Test Cases, MIP

\section{Notation}

\section{Sets}

$\mathscr{N} \quad$ Set of buses.

$\mathscr{L} \quad$ Set of branches.

$\mathscr{F}_{n} \quad$ Set of branches with bus $n$ defined as the from bus.

$\mathscr{T}_{n} \quad$ Set of branches with bus $n$ defined as the to bus.

$\mathscr{H} \quad$ Set of zones.

$\mathscr{B} \quad$ Set of boundary nodes.

$\mathscr{E} \quad$ Set of boundary edges.

$\mathscr{D}_{1} \quad$ Set of nodes with nodal degree 1.

$\mathscr{G} \quad$ Subset of $\mathscr{N}$ with generation.

$\mathscr{D} \quad$ Subset of $\mathscr{N}$ with load.

\section{Parameters}

$\delta_{\max } \quad$ Maximum allowable angle difference between adjacent nodes.

$f_{\max } \quad$ Maximum allowable branch flow.

$P_{g / d}^{\max / m i n}$ Maximum and minimum allowable generation and load, respectively, at a single bus.

$P_{g / d} \quad$ Power generation and demand respectively at a given bus.

$b \quad$ Branch susceptances.

$p \quad$ Nodal power injections: $p_{n}=P_{g, n}-P_{d, n}$.

$\mathcal{M}$ Large multiplier used to create disjunctive constraints.

$\nu, \omega \quad$ Dual variables in Lagrange relaxation and ADMM respectively. $\alpha[t], \rho[t]$ Dual variable update step size for iteration $t$ in Lagrange relaxation and ADMM respectively.

\section{Variables}

\begin{tabular}{l|l}
$\theta$ & $|\mathscr{N}| \times 1$ nodal angles.
\end{tabular}

$s \quad|\mathscr{L}| \times 1$ absolute value of angle difference across a branch.

$f \quad|\mathscr{L}| \times 1$ branch power flows.

$\beta \quad|\mathscr{E}| \times 1$ flows on inter-zone branches.

$\Pi \quad|\mathscr{N}| \times|\mathscr{N}|$ power injection permutation matrix.

$Z \quad|\mathscr{L}| \times|\mathscr{L}|$ branch susceptance permutation matrix.

$\alpha_{p / d} \quad|\mathscr{N}| \times 1$ scaling variable in range $[0,1]$ for generation $g$ and load $d$ respectively.

Given a vector $x$ and a set $\mathscr{A}, x_{\mathscr{A}}$ is defined as the subvector composed of the indices of $x$ that are in $\mathscr{A}$ :

$$
x_{\mathscr{A}} \triangleq\left\{x_{i}: i \in \mathscr{A}\right\} .
$$

Subscripts are also used to index variables by their respective sets, so that $f_{\ell}$ is the flow on line $\ell \in \mathscr{L}$. Edges are either referred to by an index, as in $\ell \in \mathscr{L}$, or by the node pair defining the edge, $(f, t) \in \mathscr{L}$. When both index and node pair are needed, we notate it as $\ell:(f, t) \in \mathscr{L}$, meaning that $f$ and $t$ are the end nodes of edge $\ell$.

\section{Introduction}

The general problem considered in this paper is how to assign properties to a topology, i.e., collection of nodes and edges of a graph. More specifically, the graph considered represents a bulk transmission power system, such that the DC powerflow assumptions [1], [2] are valid. The goal can therefore be translated to assigning the key properties in the DC powerflow-power injections $p$, and susceptances $b$ to a topology, in such a way that the resulting case will exhibit reasonable behavior. The most natural elements to look at when considering how reasonable a DC powerflow solution is, are the angle differences between adjacent nodes and the real power flowing in the branches. Empirically, (see Section 5) the distribution of angle difference appears to fit a Laplacian distribution well, which also serves to motivate the one norm minimization objective in the main formulation.

URI: http://hdl.handle.net/10125/50235

ISBN: 978-0-9981331-1-9 


\subsection{Motivation and Related Work}

This work is motivated by growing sentiment in the power systems research community that there is a lack of publicly available test cases [3]-[5]. As a result, it is particularly difficult to reproduce many results in the literature. Additionally, easier access to larger number of cases enables more robust testing on ensembles of test systems à la Monte Carlo methods.

The topology of the power grid has been studied quite extensively in the literature. It was first compared to smallworld graphs in [6], then to preferential attachment models in [7], and many others since, a review of which can be found in [8]. Other work has built on top of these studies to synthesize topologies. In [3] small world graphs are used as the backbone for construction, while [9] uses a growth model more similar to preferential attachment, and [10] exploits clustering related observations. While these works focus on topology, most of them treat the properties that attach to the topology either briefly, as in [3], or not at all.

A good fit of some structural features of the admittance matrix is insufficient for power systems analysis, because to solve a simple power flow, the additional basic information placing load and generation on the graph is needed. One can easily draw the values of generator and load set-points from realistic distributions, but a completely random assignment will, in general, lead to power flow solutions that differ significantly from realistic ones.

More recent work has emphasized that the assignment problem is far from trivial. In [11] a correlation between the system impedance matrix and location of generators is observed, and [12], [13] explores some of the coupling phenomena between different bus types in the power system. The authors in [13] used their finding to solve the placement problem for generation and load so that the statistical features found in real cases regarding the relationship between degree and generation and load are matched.

Here we look at the problem from another perspective. The main problem with placing the generation, loads and impedance at random is that it results in unrealistic operating points for the grid sample, making the synthetic case useless for any kind of realistic analysis. This motivation is supported by the numerical analysis carried out in Section 5.2. Rather than trying to fit some structural feature that may or may not be directly impacting the power flow solutions, our idea is that one can directly reproduce likely operating points statistics as the objective of the placement problem, starting from a topology and assigning load, generation and impedances through an optimization problem. In short, the novelty of this approach is that, rather than trying to fit statistics of structural properties we are directly targeting the statistics of the operational values.

It should be noted that there is another current approach to building large synthetic systems, as exemplified in [4], where geographic and other publicly available data is utilized to construct systems on a geographical footprint. The approach in [4] is therefore capable of providing far richer cases, in terms of included parameters, at the cost of currently requiring more fine-tuning by hand.

\subsection{Problem Scope}

This paper attempts to find a somewhat agnostic compromise between all the previously mentioned works. The problem is formulated as follows:

Given a topology, set of power injections, and line susceptances of appropriate size, find a suitable assignment for the elements of the two sets resulting in satisfactory DC powerflow results.

The criterion that we choose to define the suitability of the assignment is that the solution of the DC power flow problem produces a realistic distribution of phase angle differences. Since the topology as well as the distributions of power injections, $p$, and branch susceptances, $b$, are assumed correct, the specific method in which they are generated is apart from the assignment method. The main novelty of the work is to provide an automatic way of generating usable powerflow cases given these inputs, with the capability of generating an arbitrary number of cases just as easily as it produces a single one, neglecting the effects of computation time.

The remainder of the paper is structured as follows. An extensive formulation of the problem is presented in Secton 3. Since this problem quickly becomes impossibly large, Section 4 introduces methods to decompose the problem into independent, smaller, and computationally manageable subproblems. Section 5 reports results from experiments and Section 6 provides further discussion of these results and highlights the main remaining challenges. Finally, Section 7 discusses future research directions and concludes the paper.

\section{Problem Formulation}

The following inputs are assumed to be known:

1) The system topology, where each edge has a defined from and to bus.

2) Set of nodal injections $p$, but without mapping to the topology.

3) Set of branch susceptances $b$, but without mapping to the topology.

4) Maximum allowable angle difference $\delta_{\max }$, as well as absolute maximum branch flow, $f_{\max }$.

Given these inputs, the assignment problem is solved by determining the entries of permutation matrices $\Pi$ and $Z$, which map the elements of $p$ and $b$, respectively, to the nodes and edges of the topology.

At its core, this is a feasibility problem, since the goal is to find $a$ viable solution rather than the solution. However, the solution space is, at least in theory, very large and not equally weighted, meaning that some solutions are "better" than others. Better should be understood in this context as more closely matching results seen in real cases. In keeping with observations, as well as typical power system 
operations, the objective function is chosen to minimize the angle difference magnitude between adjacent buses in the system. This choice can can be seen as simultaneously maximizing stability margins and minimizing system losses. Let us denote $s$ the absolute value of angle differences between adjacent nodes, i.e. if $(f, t)$ are end nodes of an edge $\ell$ then $s_{\ell}=\left|\theta_{f}-\theta_{t}\right|$. Our objective can be cast into the following mathematical formulation.

$$
\begin{aligned}
\underset{\Pi, Z, \theta, s, f}{\text { Minimize }} & \sum_{\ell \in \mathscr{L}} s_{\ell} \\
\text { Subject to } & -f_{\max } \leq f_{\ell} \leq f_{\max } \quad \forall \ell \in \mathscr{L} \\
& -\delta_{\max } \leq \theta_{f}-\theta_{t} \leq \delta_{\max } \quad \forall(f, t) \in \mathscr{L} \\
& f_{\ell}+b_{\tilde{\ell}}\left(\theta_{f}-\theta_{t}\right)+\left(1-Z_{\tilde{\ell}) \mathcal{M} \geq 0}\right. \\
& \forall \ell:(f, t), \tilde{\tilde{\ell}} \in \mathscr{L} \\
& f_{\ell}+b_{\tilde{\ell}}\left(\theta_{f}-\theta_{t}\right)-\left(1-Z_{\tilde{\ell}) \mathcal{M} \leq 0}\right. \\
& \sum_{\tilde{n} \in \mathscr{N}} p_{\tilde{n}} \Pi_{n \tilde{n}}+\sum_{\ell \in \mathscr{T}_{n}} f_{\ell}-\sum_{\ell \in \mathscr{F}_{n}} f_{\ell}=0 \quad \forall n \\
& \sum_{\tilde{n} \in \mathscr{N}} \Pi_{n \tilde{n}}=1, \sum_{\tilde{n} \in \mathscr{N}} \Pi_{\tilde{n} n}=1 \quad \forall n \in \mathscr{N} \\
& \sum_{\tilde{\ell} \in \mathscr{L}} Z_{\ell \tilde{\ell}}=1, \sum_{\tilde{\ell} \in \mathscr{L}} Z_{\tilde{\ell} \ell}=1 \quad \forall \ell \in \mathscr{L} \\
& \Pi_{n, \tilde{n}}=0 \quad \forall n, \tilde{n}: n \in \mathscr{D}_{1} \cap p_{\tilde{n}}=0 \\
& s_{\ell}+\left(\theta_{f}-\theta_{t}\right) \geq 0 \quad \forall \ell:(f, t) \in \mathscr{L} \\
& s_{\ell}-\left(\theta_{f}-\theta_{t}\right) \geq 0 \quad \forall \ell:(f, t) \in \mathscr{L} \\
& s_{\ell} \geq 0 \quad \forall \ell \in \mathscr{L} \\
& \Pi_{n \tilde{n}} \in\{0,1\} \quad \forall n, \tilde{n} \\
& Z_{\ell \tilde{\ell}} \in\{0,1\} \quad \forall \ell, \tilde{\ell}
\end{aligned}
$$

Constraints (1b) and (1c) limit the allowable flows and angle differences, while constraints (1d)-(1f) are the DC powerflow equations. The disjunctive formulation in (1d) and (1e) is needed to select entries from susceptances, $b$, based on $Z$, without forming bilinear terms by multiplying $Z b\left(\theta_{f}-\theta_{t}\right)$ directly. Constraints $(1 \mathrm{~g})$ and $(1 \mathrm{~h})$, in conjunction with the binary constraints, force $\Pi$ and $Z$ to be permutation matrices, and constraints (1j)-(11) are simply a way to enforce $s=\left|\theta_{f}-\theta_{t}\right|$. Additionally, constraint (1i) is added to prevent leaf nodes (those with degree one) from receiving a zero injection assignment. The reasoning for this decision is that a leaf node with zero injection can be trivially removed from the system and therefore, such an assignment is undesirable.

The disjunctive constraints are bound to slow down the optimization, so it is critical to consider an appropriate $\mathcal{M}$ value. By considering (1b)-(1e), It is clear that any $\mathcal{M}$ satisfying,

$$
\mathcal{M} \geq f_{\max }+\delta_{\max } \max _{\ell}\left|b_{\ell}\right|
$$

will achieve the desired result, with equality representing the smallest possible value.

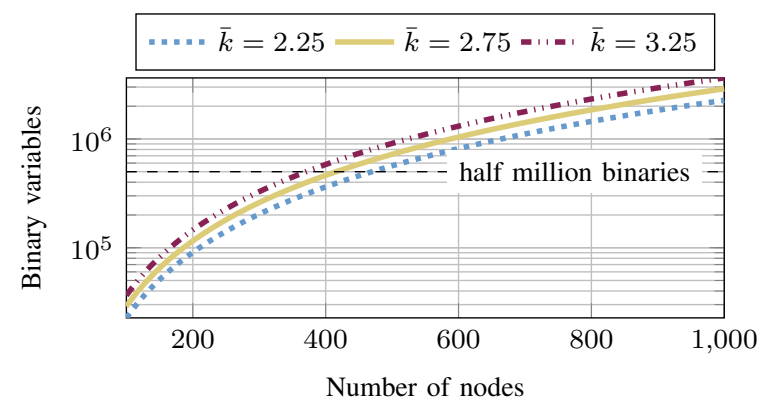

Figure 1. Relationship between topology and number of binary variables. For average nodal degrees in the range of typical power systems the number of binaries reaches half a million when the there are around 400 nodes in the system.

\section{Decompositions}

Due to the two matrices of binary variables, the problem in (1) suffers greatly from dimensionality and scales quite poorly. A large system with thousands of buses and branches translates to millions of binary variables. There are $(\bar{k} / 2) \times$ $|\mathscr{N}|$ edges in a graph with average nodal degree $\bar{k}$ and $|\mathscr{N}|$ nodes. Therefore, the total number of binary variables is $\left(1+\bar{k}^{2} / 4\right)|\mathscr{N}|^{2}$.

Figure 1 shows how the number of binary variables grows with the size of $\mathscr{N}$ in the range of typical $\bar{k}$ for power systems. Since MIP problems with millions of binaries are still too difficult to solve, a decomposition approach is necessary. The proposed decomposition approach is outlined in Figure 2 and explained in detail in the following.

\subsection{Zone Formation}

The topology is broken into smaller zones that are desired to be as independent from one another as possible. Segmenting the power system into semi-independent regions is similar in spirit to real world structure of balancing authorities $^{1}$. In cases where the balancing zones are known, they might serve as the actual computation zones. However, in the general case considered here, boundaries are unknown, and furthermore, a single balancing authority may still be too large from a computational perspective, and would therefore need to be further segmented. A method is therefore needed to split the topology, which amounts to solving a graph partitioning problem. The Algebraic Connectivity, $\lambda_{2}$, and the corresponding Fiedler vector [14] are used to repeatedly split up zones until no zone is larger than a given maximum. The procedure is detailed in the algorithm in Figure 3.

All the procedure in Figure 3 does is split graphs into two parts based on the sign of the Fiedler vector elements. As stated in [15], this has been shown to be a good heuristic for partitioning graphs with small interference, i.e., few edges between the two partitions. It is possible that after

1. An illustrative example for WECC can be found here: https://www. wecc.biz/Administrative/WECC_BAMap.pdf 


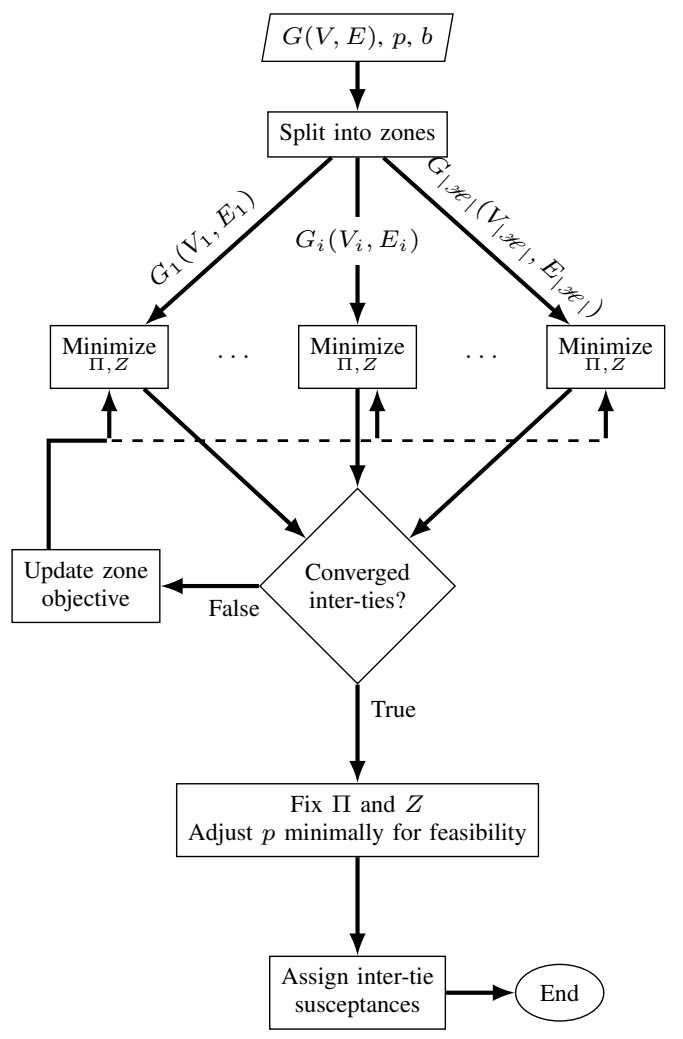

Figure 2. Flowchart for solution via zonal decomposition.

multiple iterations the induced subgraphs will become disconnected. For this reason, the second half of the algorithm in Figure 3 checks for disconnected components, and if any are smaller than a predefined size, $N_{\min }$, it finds a neighboring subgraph and combines the two. This procedure could be of interest beyond the scope of this work, as it provides a systematic way of creating zones. Synthetic systems, regardless of how they were created, could use this approach to identify natural borders for different balancing authorities.

After zones are created, an appropriately sized subset of $p$ and $b$ are randomly assigned to each. The only restriction placed on the random assignment is that the zonal imbalance, i.e., the sum of the subset of $p$ in absolute value, be smaller than the maximum import/export capacity for the zone. This is in turn dictated by the number of boundary edges and the maximum allowable flow. For example, for zone $i$ the following would have to hold:

$$
\left|\sum_{n \in \mathscr{H}_{i}} p_{n}\right| \leq f_{\max }\left|\mathscr{E}_{i}\right| .
$$

This initial assignment greatly simplifies the problem but has the implication that the decomposition cannot be completely equivalent to the extensive formulation. However, for sufficiently large zones, the difference should be minimal. Furthermore, achieving optimality is not really necessary to obtain good statistics (see also Section 5).

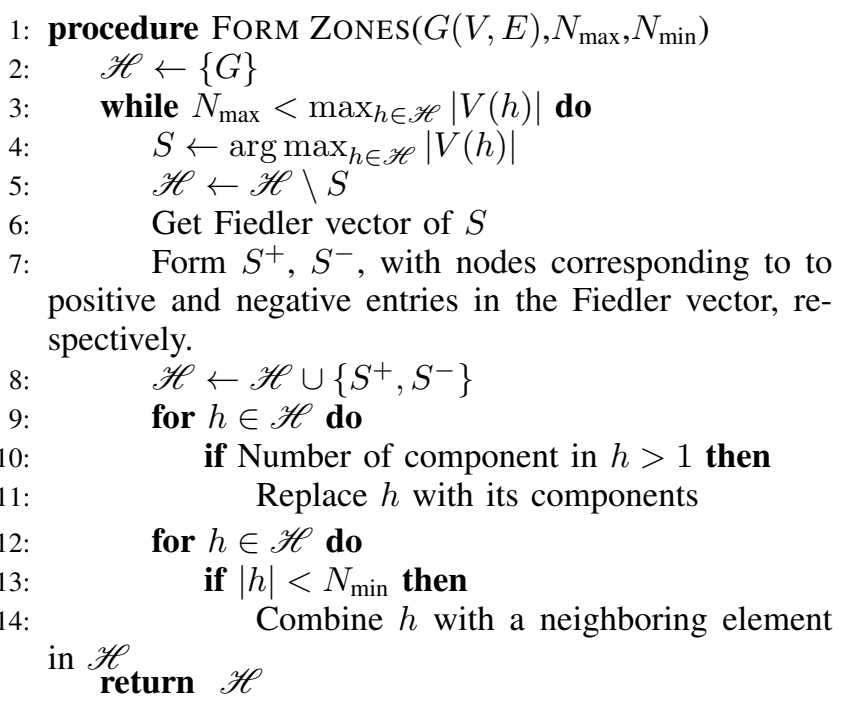

Figure 3. Algorithm for splitting transmission grid graph topology into zones.

While zone partitioning assigns each node to exactly one subproblem, the boundary edges are dropped. As shown in Figure 2, the remaining inter-tie susceptances are assigned at the end of the procedure based on the final flow on each boundary edge. The largest flows get the largest magnitude (most negative) susceptances, thereby minimizing the expected angle differences as much as possible.

\subsection{Inter-Zone Flows}

Nodes on the boundary of zones are now capable of receiving and sending power on edges that are not represented in their subproblem. To handle this discrepancey, balance constraints (1f) for the boundary nodes are relaxed by adding slack variables corresponding to inter-tie flows, identified as $\beta$. Figure 4 illustrates the basic relationships between zones expressed in the equations that follow. Constraint (1f) for boundary nodes becomes,

$$
\begin{aligned}
& \sum_{\tilde{n}} p_{\tilde{n}} \Pi_{n \tilde{n}}+\sum_{\ell \in \mathscr{T}_{n}} f_{\ell}-\sum_{\ell \in \mathscr{F}_{n}} f_{\ell}+ \\
& \sum_{\ell \in \mathscr{E}_{i} \cap \mathscr{T}_{n}} \beta_{\ell}^{i}-\sum_{\ell \in \mathscr{E}_{i} \cap \mathscr{F}_{n}} \beta_{\ell}^{i}=0, \quad \forall i \in \mathscr{H}, \forall n \in \mathscr{B}_{i}
\end{aligned}
$$

where $\mathscr{B}_{i}$ is the set of boundary nodes for zone $i$, and $\mathscr{E}_{i}$ the set of boundary edges for zone $i$. To minimize the absolute value of inter-tie flows, the following constraints are added:

$$
\begin{array}{ll}
\beta_{\ell}^{i,+} \geq \beta_{\ell}^{i} & \forall i \in \mathscr{H}, \forall \ell \in \mathscr{E}_{i} \\
\beta_{\ell}^{i,-} \geq-\beta_{\ell}^{i} & \forall i \in \mathscr{H}, \forall \ell \in \mathscr{E}_{i}
\end{array}
$$

and the objective becomes,

$$
f\left(s, \beta^{+}, \beta^{-}\right)=\sum_{\ell \in \mathscr{L}} s_{\ell}+\sum_{\ell \in \mathscr{E}}\left(\beta_{\ell}^{+}+\beta_{\ell}^{-}\right) .
$$




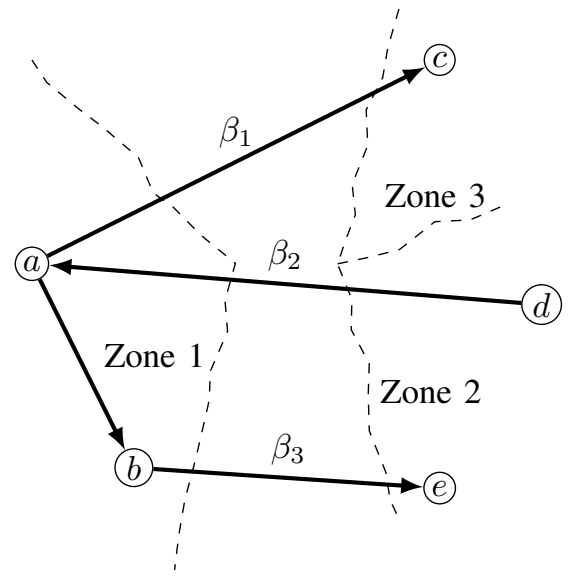

Figure 4. Illustration of the inter-zone flows. Using the defined notation, $\mathscr{E}_{1,2}=\{2,3\}, \beta_{\mathscr{E}_{1,3}}^{3}=\left[\beta_{1}^{3}\right], \mathscr{E}_{1} \cap \mathscr{T}_{a}=\{2\}$, and $\mathscr{E}_{1} \cap \mathscr{F}_{a}=\{1\}$.

By defining the sets of edges between any two zones $i$ and $j$ as,

$$
\mathscr{E}_{i j}= \begin{cases}\mathscr{E}_{i} \cap \mathscr{E}_{j} & i<j \\ \emptyset & \text { otherwise }\end{cases}
$$

the constraints coupling the zones can be expressed as,

$$
\beta_{\ell}^{i}=\beta_{\ell}^{j} \quad \forall \ell \in \mathscr{E}_{i j}, \forall i, j \in \mathscr{H},
$$

which simply mean that the zones agree on the inter-tie flow. The full problem can therefore be reformulated $t^{2}$ :

$$
\begin{array}{cl}
\text { Minimize } & \sum_{i \in \mathscr{H}} f_{i}\left(s_{i}, \beta_{i}\right) \\
\text { Subject to } & \beta_{\ell}^{i}=\beta_{\ell}^{j} \quad \forall \ell \in \mathscr{E}_{i j}, \forall i, j \in \mathscr{H} \\
& \text { constraints in (1), (4), and (5). }
\end{array}
$$

Since the constraints in (8) are the only ones with "complicating" variables, that is, they are the only ones coupling the zones, they are the obvious choice for relaxation, which is considered in the next two sections.

\subsection{Solution by Lagrange Relaxation}

Recalling that the subvector of $\beta_{i}$ corresponding to the boundary between zones $i$ and $j$ is denoted as,

$$
\beta_{\mathscr{E}_{i j}}^{i}=\left\{\beta_{\ell}^{i}: \ell \in \mathscr{E}_{i j}\right\},
$$

the Lagrangian for the problem in (9) with (8) relaxed is,

$$
\begin{aligned}
& L\left(s_{1}, \ldots, s_{|\mathscr{H}|}, \beta_{1}, \ldots, \beta_{|\mathscr{H}|}\right)= \\
& \sum_{i \in \mathscr{H}} f_{i}\left(s_{i}, \beta_{i}\right)+\sum_{i, j \in \mathscr{H}} \nu_{\mathscr{E}_{i j}}^{T}\left(\beta_{\mathscr{E}_{i j}}^{i}-\beta_{\mathscr{E}_{i j}}^{j}\right) .
\end{aligned}
$$

2. $\beta^{ \pm}$are replaced by $\beta$ for notational clarity, since they are only a way of representing the absolute value of $\beta$.
Each subproblem or zone, $k$, can be now solved separately:

$$
\text { Minimize } f\left(s_{k}, \beta_{k}\right)+\sum_{j \in \mathscr{H}} \nu_{\mathscr{E}_{k j}}^{T} \beta_{\mathscr{E}_{k j}}^{k}-\sum_{i \in \mathscr{H}} \nu_{\mathscr{E}_{i k}}^{T} \beta_{\mathscr{E}_{i k}}^{k}
$$

Subject to constraints in (1), (4), and (5) for zone $k$ only.

Dual variable vector $\nu$ is updated after each iteration $t$ based on the rule:

$$
\nu_{\mathscr{E}_{i j}}[t+1]:=\nu_{\mathscr{E}_{i j}}[t]+\alpha[t]\left(\beta_{\mathscr{E}_{i j}}^{i}[t]-\beta_{\mathscr{E}_{i j}}^{j}[t]\right) \quad \forall \mathscr{E}_{i j}
$$

where $\alpha[t]$ is a chosen step size. The dual in this way can be seen to represent the "price" of enforcing equality constraint (8), or conversely the benefit of relaxing it with some slack.

Due to the non convex nature of each subproblem, the usual guarantees on dual decomposition and gradient decent do not hold. In practice, the problem is found to be highly sensitive to step size $\alpha[t]$. Without very careful tuning, the solution oscillates, so that every element in $\beta$ that is in disagreement with its neighbor alternates sign on each iteration. To address this issue, an alternative decomposition routine is desired.

\subsection{Solution by ADMM}

Robustness is added to the solution via an augmented Lagrangian. The Alternating Direction Method of Multipliers (ADMM) as described in [16] is adopted, where $\beta$ is the communicated variable. This approach is also very similar to the Progressive Hedging method as described in [17]. Instead of updating the dual variable based on the difference between the copies of each element in $\beta$ as done in (13), the update is with respect to the average value,

$\omega_{\mathscr{E}_{i j}}^{k}[t+1]:=\omega_{\mathscr{E}_{i j}}^{k}[t]+\rho[t]\left(\beta_{\mathscr{E}_{i j}}^{k}[t]-\bar{\beta}_{\mathscr{E}_{i j}}[t]\right) \quad \forall \mathscr{E}_{i j}, k \in i, j$,

where,

$$
\bar{\beta}_{\mathscr{E}_{i j}}[t]=\frac{\beta_{\mathscr{E}_{i j}}^{i}[t]+\beta_{\mathscr{E}_{i j}}^{j}[t]}{2} .
$$

Note that the dual variable $\omega$ is now local to each zone, as opposed to $\nu$, which is global.

In addition to the slightly altered dual update, the augmented Lagrangian adds a quadratic term to the objective. and the new the problem in each zone, $k$ becomes:

$$
\begin{aligned}
\text { Minimize } & f\left(s_{k}, \beta_{k}^{+}, \beta_{k}^{-}\right)+\sum_{j \in \mathscr{H}}\left(\omega_{\mathscr{E}_{k j}}^{k}\right)^{T} \beta_{\mathscr{E}_{k j}}^{k} \\
+ & \sum_{i \in \mathscr{H}}\left(\omega_{\mathscr{E}_{i k}}^{k}\right)^{T} \beta_{\mathscr{E}_{i k}}^{k}+\frac{\rho}{2}\left\|\beta^{k}-\bar{\beta}_{\mathscr{E}_{k}}\right\|_{2}^{2}
\end{aligned}
$$

Subject to constraints in (1), (4), and (5) for zone $k$ only.

The main advantage of the ADMM decomposition over the simpler and linear Lagrange relaxation, is that a penalty on a specific distance from a value is possible, which helps prevent oscillations. To achieve the same results without resorting to a quadratic term, constraints similar to (5) are necessary, which alter both of the feasible region, as well as the objective, and introduce more variables. 


\subsection{Forcing Consensus}

Due to the non-convex nature of the problem, the zones are not likely to fully converge to a consensus on $\beta$. This discrepancy can be significant enough that the final DC powerflow will violate some of the initial constraints, specifically, the flow or angle difference limits.

To resolve this issue, a final iteration is performed where $\Pi$ and $Z$ are fixed, meaning that the power injections and suceptances are now stricktly parameters of the problem. As such, the problem is convex and therefore, the entire system can be handled instead of the individual zones. Two new variables, $\alpha_{g}$ and $\alpha_{d}$ are introduced, which scale each generation and load entry. The goal of the forced consensus problem is to alter the the power injection vector as little as possible, while still satisfying all the constraints. This is achieved by a the quadratic objective in the (17). Since the generation and load are now handled individually, instead of parameter $p$ we have the nodal generation $P_{g}$ and nodal consumption $P_{d}$. We also use sets $\mathscr{G}$ and $\mathscr{D}$ to describe the non-zero entries of $P_{g}$ and $P_{d}$ respectively.

$$
\begin{array}{cl}
\underset{\alpha_{g}, \alpha_{d}, f, \theta}{\text { Minimize }} & \sum_{n \in \mathscr{G}}\left(\alpha_{g, n}-1\right)^{2}+\sum_{n \in \mathscr{D}}\left(\alpha_{d, n}-1\right)^{2} \\
\text { Subject to } & f_{\ell}+b_{\ell}\left(\theta_{f}-\theta_{t}\right)=0 \quad \forall \ell \in \mathscr{L} \\
& \left(\alpha_{g, n} P_{g, n}-\alpha_{d, n} P_{d, n}\right)+\sum_{\ell \in \mathscr{T}_{n}} f_{\ell}+ \\
& -\sum_{\ell \in \mathscr{F}_{n}} f_{\ell}=0 \quad \forall n \in \mathscr{N} \\
& -f_{\max } \leq f_{\ell} \leq f_{\max } \quad \forall \ell \in \mathscr{L} \\
& -\delta_{\max } \leq \theta_{f}-\theta_{t} \leq \delta_{\max } \quad \forall(f, t) \in \mathscr{L} \\
& P_{g}^{\min } \leq \alpha_{g, n} P_{g, n} \leq P_{g, \max } \quad \forall n \in \mathscr{G} \\
& P_{d}^{\min } \leq \alpha_{d, n} P_{d, n} \leq P_{d, \max } \quad \forall n \in \mathscr{D} \quad(17 \mathrm{~g}) \\
& \alpha_{d, n} P_{d, n} \leq \alpha_{g, n} P_{g, n} \\
& \forall n \in \mathscr{G} \cap \mathscr{D}: P_{d, n}<P_{g, n} \\
& \alpha_{d, n} P_{d, n} \geq \alpha_{g, n} P_{g, n} \\
& \forall n \in \mathscr{G} \cap \mathscr{D}: P_{d, n}>P_{g, n} \\
& \alpha_{g}, \alpha_{d} \geq 0
\end{array}
$$

Constraints (17b) and (17d) are the DC powerflow formulation where the injection is explicitly shown as the difference between load and generation. To avoid unreasonable numbers, limits are placed on the both load and generation by constraints (17f) and (17g). Finally, constriants (17i) and $(17 \mathrm{j})$ ensure that the relative relation between load and generation on a bus that has both does not change.

\section{Results}

Our procedure can be paired with a topology generation algorithm but can also be used simply to complete and/or reshuffle a real case. For testing, the Polish2383wp case available in Matpower [18] is used as a reference case. In one test, the data from the case is simply reshuffled, while in a second one distributions are extracted and used to generate input sets that are placed on an RT-nested-SmallWorld [3] synthetic topology. Implementation is done using the Gurobi $^{3}$ solver with Python API. The Polish2383wp case is chosen because it is publicly available, represents a real system, and is sufficiently large that decomposition is absolutely necessary. With an average degree of 2.43 , the full problem has over 14 million binary variables.

\subsection{Input Data}

As stated in Section 3, an edge list, as well as a set of power injections, and line susceptances are needed as inputs to the algorithm. We do not discuss appropriate ways to generate topologies but rather assume that the provided topology is satisfactory.

For the sake of comparison, the distributions of generation, load, and reactance should be similar to the reference case. This is achieved by fitting the empirical cumulative distribution functions from the reference case with a Piecewise Cubic Hermite Interpolating Polynomial (PCHIP) [19]. Since this interpolation method is monotone it provides a valid cumulative distribution. At the same time, the result is smoother than an empirical histogram.

In addition to the distributions the following quantities are necessary:

$N_{\text {int }} \quad$ The number of intermediate buses with nei-

ther load nor generation.

$N_{g} \quad$ Number of buses with generation.

$N_{\text {gen only }} \quad$ Number of buses with only generation.

$N_{P_{d}<P_{g}} \quad$ Number of of buses with generation and load where generation is greater.

These can be calculated from fractions extracted from the reference case and the total number of buses in the cases that is being constructed. The procedure for sampling generation $P_{g}$, and load $P_{d}$, (and therefore power injection) data is as follows:

1) $\quad N_{\text {int }}$ entries are set to zero for both $P_{g}$ and $P_{d}$.

2) $\quad N_{\text {load only }}=|\mathscr{N}|-\left(N_{\text {int }}+N_{g}\right)$ load only buses are sampled for the load distribution, corresponding entries in $P_{g}$ are zero.

3) $\quad N_{\text {gen only }}$ buses are sampled from the generation distribution, corresponding entries in $P_{d}$ are zero.

4) For each of the $N_{P_{d}<P_{g}}$ entries, generation is sampled first. Then load samples are drawn until one is found that is smaller than the sampled generation.

5) For each of the $N_{P_{d}>P_{g}}=N_{g}-\left(N_{\text {gen only }}+\right.$ $\left.N_{P_{d}<P_{g}}\right)$ entries, load is sampled first. Then generation samples are drawn until the inequality is satisfied.

The load and generation sampled from their respective distributions will likely not equal. For the simple DCpowerflow neglecting losses, this should be remedied. This

3. http://www.gurobi.com/ 
is done using the optimization in (18), which is very similar to (17).

$$
\begin{array}{cc}
\underset{\alpha_{g}, \alpha_{d}}{\operatorname{Minimize}} & \sum_{n \in \mathscr{G}} \frac{P_{g, n}}{\max \left(P_{g}\right)}\left(\alpha_{g, n}-1\right)^{2}+ \\
& \quad+\sum_{n \in \mathscr{D}} \frac{P_{d, n}}{\max \left(P_{d}\right)}\left(\alpha_{d, n}-1\right)^{2} \\
\text { Subject to } & \sum_{n \in \mathscr{G}} \alpha_{g, n} P_{g, n}-\sum_{n i \in \mathscr{D}} \alpha_{d, n} P_{d, n}=0 \\
& P_{g}^{\min } \leq \alpha_{g, n} P_{g, n} \leq P_{g, \max } \quad \forall n \in \mathscr{G} \\
& P_{d}^{\min } \leq \alpha_{d, n} P_{d, n} \leq P_{d, \max } \quad \forall n \in \mathscr{D} \\
& \alpha_{d, n} P_{d, n} \leq \alpha_{g, n} P_{g, n} \\
& \forall n \in \mathscr{G} \cap \mathscr{D}: P_{d, n}<P_{g, n} \\
& \alpha_{d, n} P_{d, n} \geq \alpha_{g, n} P_{g, n} \\
& \forall n \in \mathscr{G} \cap \mathscr{D}: P_{d, n}>P_{g, n} \\
\alpha_{g}, \alpha_{d} \geq 0
\end{array}
$$

The optimization finds multipliers for each entry of $P_{g}$ and $P_{d}$ such that the total load and generation will be equal. The objective attempts to keep these multipliers as close to one as possible, thus preserving the shape of the sampled distribution. Additionally, each term in the objective is weighted with respect to the largest generation or load, which rewards the program for preserving the tails, i.e., prevent it from altering the few large generators or loads too strongly.

Figure 5 shows the distributions of the real case, the original drawn samples, and final input samples to the algorithm following optimization (18). It is evident that the samples are fairly similar in distribution, and are furthermore not greatly affected by the modification.

\subsection{Motivation for Optimization: Random Assign- ment}

To motivate the need for applying (1) (or its subsequent decomposition), we first present what happens if the assignment is performed uniformly at random. Power injections and impedance are sampled as described in Section 5.1 and assigned by picking uniformly and independently a bus (for load and generation) or a line (for admittances) from the 3000 node RT-nested-smallWorld topology [3]. The process is repeated 400 times, and for each the maximum angle difference and line flow is recorded. Figure 6 shows the distributions of these maximum values. Note that some of the maximum angle differences in Figure 6a are greater than $90^{\circ}$, which is non-sensical from a physical perspective. The frequent occurrence of unacceptably large extreme values reinforces the idea that some form of optimization is necessary to meet the requirements of a realistic operating point.

\subsection{Experiment Setup}

Two experiments are considered in comparison to the reference case:

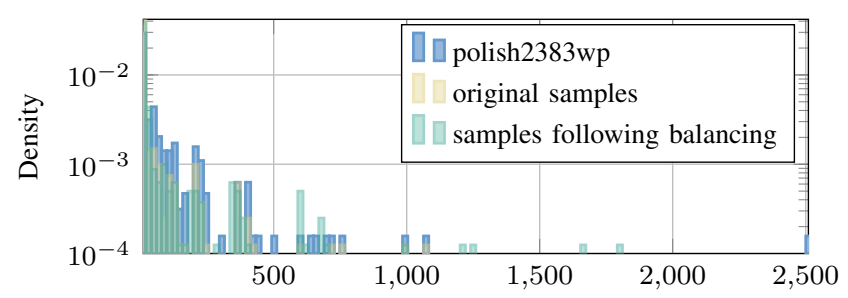

(a) $P_{g}[\mathrm{MW}]$

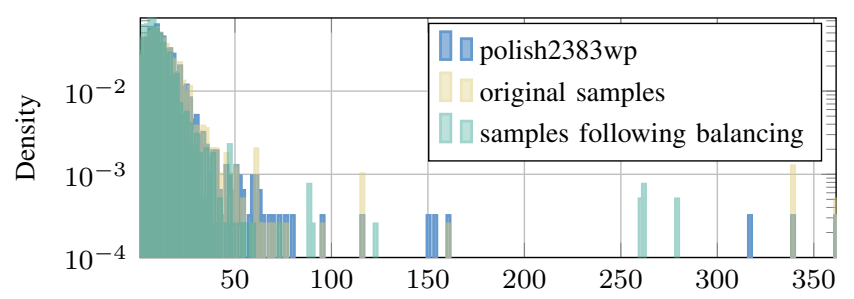

(b) $P_{d}[\mathrm{MW}]$

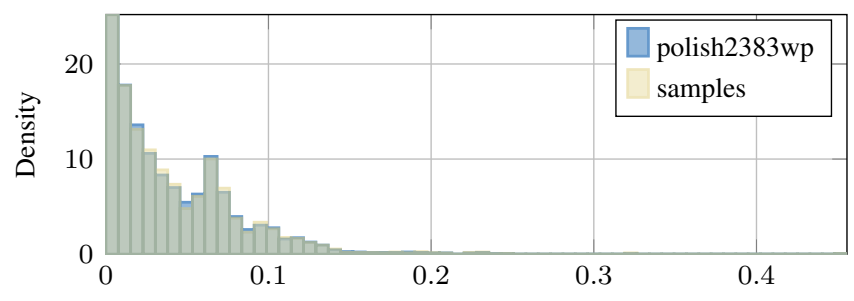

(c) $x$ [p.u.]

Figure 5. Input distributions for (a) generation, (b) load, and (c) impedance, showing the data from the original polish2383wp case and the drawn samples for placement on the RT-nested-SmallWorld 3000 topology. Figures (a) and (b) also show the (relatively minimal) effect of the balancing optimization in (18).

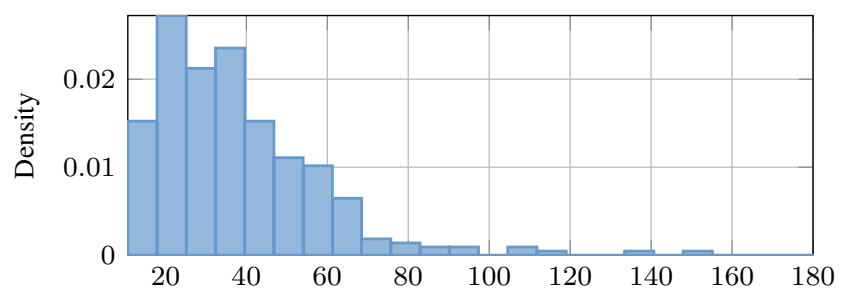

(a) Maximum $\theta_{f}-\theta_{t}$ [degrees]

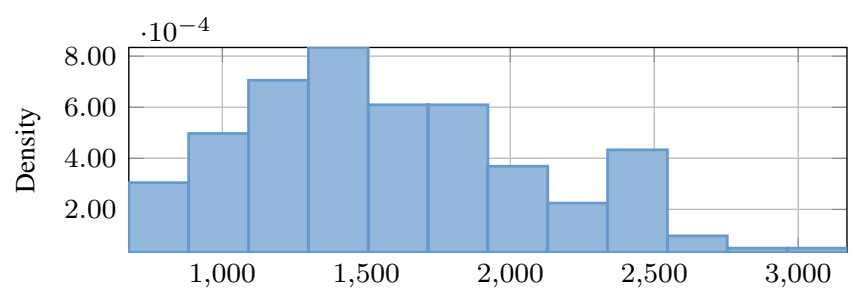

(b) Maximum Line Flow [MW]

Figure 6. Distribution of (a) maximum angle difference and (b) maximum line flow, over 400 RT-nested-smallWorld 3000 cases where power injection and impedances are assigned uniformly at random. The frequent occurrence of unacceptably large values motivates the investigation of more complex placement processes. 


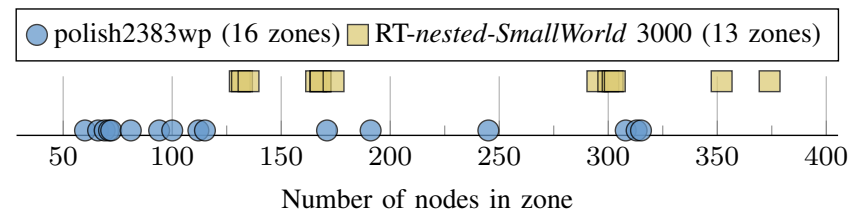

Figure 7. Sizes of zones after partitioning using the algorithm in Figure 3.

1) Polish2383wp reassigned comprises the same topology, power injections $p$, and line susceptances $b$, as the reference case. The optimization routine is tasked with finding a suitable mapping for $p$ and $b$.

2) RT-nested-SmallWorld $\mathbf{3 0 0 0}$ is a fully synthetic sample. The topology is generated using the RTnested-SmallWorld algorithm in [3], and sets $p$ and $b$ are sampled as described in Section 5.1.

The topologies are broken up into zones with no more than 400 nodes and no fewer than 50. Figure 7 shows the resulting sizes for the zones in the two topologies. The computational difficulty of the problem is closely linked to sizes of the zones. As discussed Section 6, the largest zones pose major difficulties in achieving convergence. ADMM is used to update each zone and parameter $\rho[t]$ is set to,

$$
\rho[t]:= \begin{cases}1 / \sqrt{t} & t>0 \\ 1 & t=0 .\end{cases}
$$

on each iteration $t$. In all problems $f_{\max }$ is set to 10 p.u. and $\delta_{\max }$ to $60^{\circ}$.

At the end of every iteration the following statistics about the agreement between the $\beta$ variables are collected:

$$
\begin{aligned}
\text { Gap: } & \sum_{i \in \mathscr{H}} \sum_{\ell \in \mathscr{E}_{i}}\left|\beta_{\ell}^{i}-\bar{\beta}_{\ell}\right| \\
\text { Mean Error: } & \frac{1}{|\mathscr{E}|} \sum_{i, j \in \mathscr{H}} \sum_{\ell \in \mathscr{E}_{i j}}\left|\beta_{\ell}^{i}-\beta_{\ell}^{j}\right| \\
\text { Max Error: } & \max \left(\sum_{i, j \in \mathscr{H}} \sum_{\ell \in \mathscr{E}_{i j}}\left|\beta_{\ell}^{i}-\beta_{\ell}^{j}\right|\right) .
\end{aligned}
$$

If any fall below their respectively prescribed threshold, the optimization concludes. Additionally, each subproblem solving time is limited to 300 seconds, an a relative MIP gap stopping criteria of $15 \%$ is set. Finally, a limit of 5 iterations is imposed.

\subsection{Powerflow Results}

The results are evaluated with respect to the values associated with a DC powerflow, namely, the angle differences between adjacent buses and the real power flows. Distributions for these quantities are shown in Figure 8, which provide a strong visual indication that there is good agreement. The majority of the weight in both distributions is centered around zero, however, they exhibit heavy tails, meaning that there are non-negligible extreme values. Table 1 reports these extreme values for the three cases in Figure 8.

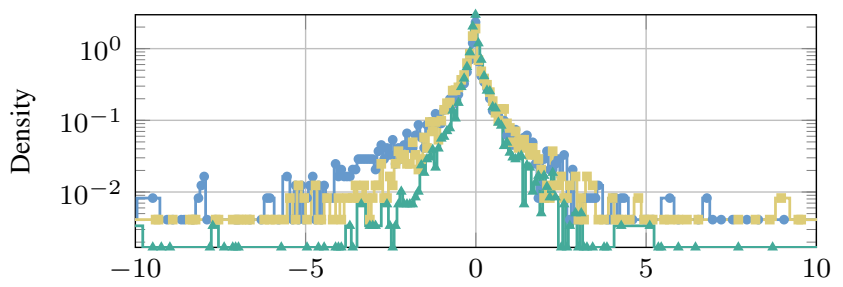

(a) $\theta_{f}-\theta_{t}$ [degrees]

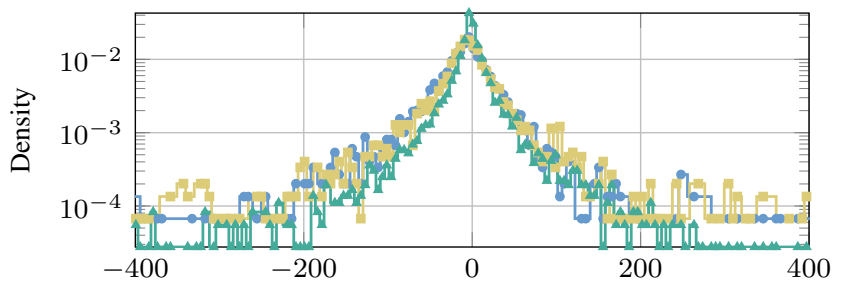

(b) Line Flow $[\mathrm{MW}]$

\begin{tabular}{ll|}
$\square$ & Polish2383wp \\
& Polish2383wp reassigned \\
& RT-nested-SmallWorld 3000
\end{tabular}

Figure 8. Two optimization results compared to the original case

TABLE 1. MAXIMUM VALUES IN RESULTS

\begin{tabular}{lcc}
\hline Case & Max Flow [MW] & Max $\Delta \theta$ [degrees] \\
\hline Polish 2383wp & 882 & 14.59 \\
Polish2383wp reassigned & 958 & 24.59 \\
RT-nested-SmallWorld 3000 & & \\
\cline { 1 - 1 } \multicolumn{1}{c}{ pre forced consensus } & 2631 & 20.08 \\
post forced consensus & 1000 & 17.46 \\
\hline
\end{tabular}

Table 1 also shows the result for the RT-nestedSmallWorld case before the forced consensus step in (17) is performed. As the maximum allowable flow was set to $1000 \mathrm{MW}$ in all the problems, there is clearly a violation. The highlights the need for the final forced consensus step. Additionally, it confirms that simple random assignment is not likely to produce satisfactory results. Since the final results meet all the constraints, while intermediate ones do not, we can conclude that the optimization is both necessary and fulfills its function of assigning the parameters in a way that leads to a viable and reasonable DC powerflow result.

\section{Discussion}

The results presented in the previous section indicate that the proposed algorithm successfully achieves its goal. A closer examination reveals a few issues that merit some further discussion. Table 2 reports the termination criteria values at the end of the optimization as well as run times. Both optimization terminated after the iteration limit of five iterations was reached.

Scalability could be rightfully questioned if for $\sim 3000$ nodes the run-time is over five hours. Additionally, a motivation for automating case generation, is to produce multiple 
TABLE 2. OPTIMIZATION RUN STATISTICS

\begin{tabular}{|c|c|c|}
\hline & $\begin{array}{l}\text { Polish2383wp } \\
\text { reassigned }\end{array}$ & $\begin{array}{l}\text { RT-nested- } \\
\text { SmallWorld } \\
3000\end{array}$ \\
\hline Total Run Time* & $5 \mathrm{hr} 20 \mathrm{~min}$ & $8 \mathrm{hr} 31 \mathrm{~min}$ \\
\hline Final Gap [p.u] & 29.249 & 80.288 \\
\hline Final Mean Error [p.u] & 0.152 & 0.328 \\
\hline Final Max Error [p.u] & 1.242 & 1.422 \\
\hline
\end{tabular}

examples, run multiple simulations, and therefore achieve more statistically meaningful results. That is, run MonteCarlo simulations varying not only parameters but actual systems. To achieve this, a large number of cases need to be generated. If each case requires multiple hours, the time requirement for a hundred could become too onerous. Time is important, therefore, not from an operation critical perspective, but rather in term of user acceptance.

Gurobi's root relaxation, performed to find a lower bound prior to the branch and bound process is the main culprit for the long computation times. The combination of a quadratic objective and disjunctive constraints (1d) and (1e) lead to a poor and slow relaxation. In combination with the time limit imposed on the optimization, this has the result that larger zones often do not complete the root-relaxation, no new solution is found, and consequently no improvement towards convergence is made.

The observed maximum error is likely a result of these computational issues. when larger errors exist between zones that are incapable of solving their root-relaxation, they cannot improve. An obvious solution is to remove completely or extend the time limit. However, this comes at the cost of longer run-times, which are already longer than desired. Potential improvements in this area are left for future work.

\section{Conclusion and Future Work}

To fully unlock the potential of synthetic power system cases, creating new cases should be fast enough that preparing several cases is not a daunting task. This paper presented an optimization approach to assigning known power injections and line suceptances to a topology and achieve realistic DC powerflow results. Results on a moderately large sample validate the approach. The solution is only possible by decomposing the problem into smaller zones and iteratively solving using the ADMM method. While the solutions are satisfactory, future work will explore ways to speed up the process to more reasonable times for potential users.

One possibility that will be considered in future work is to relax the binary constraints entirely and replace them with a combination of orthonormal constraints on $\Pi$ and $Z$, as well as heuristics to project the results back onto the space of permutation matrices. The resulting constraints cease to be linear but perhaps a more complex but continuous feasible region will be more amenable to faster solutions. In parallel, additional elements will be added to the procedure so that more complete cases can be created that also directly address the $\mathrm{AC}$ powerflow.

\section{Acknowledgements}

This work was funded in part by the Advanced Research Projects Agency- Energy (ARPA-E), U.S. Department of Energy, under Award Number DE-AR0000714. The views and opinions of authors expressed herein do not necessarily state or reflect those of the United States Government or any agency thereof.

\section{References}

[1] A. Wood, B. Wollenberg, and G. Sheblé, Power Generation, Operation, and Control, 3rd ed. Wiley, 2014.

[2] B. Stott, J. Jardim, and O. Alsac, "Dc power flow revisited," IEEE Transactions on Power Systems, vol. 24, no. 3, pp. 1290-1300, Aug 2009.

[3] Z. Wang, A. Scaglione, and R. Thomas, "Generating Statistically Correct Random Topologies for Testing Smart Grid Communication and Control Networks," IEEE Transactions on Smart Grid, vol. 1, no. 1 , pp. 28-39, June 2010.

[4] A. Birchfield, T. Xu, K. M. Gegner, K. S. Shetye, and T. J. Overbye, "Grid Structural Characteristics as Validation Criteria for Syntheti Netowrks," IEEE Transactions on Power Systems, 2016.

[5] ARPA-E Grid Data. [Online]. Available: https://arpa-e.energy.gov/ ?q=arpa-e-programs/grid-data

[6] D. J. Watts and S. H. Strogatz, "Collective dynamics of 'small-world' networks," Nature, vol. 393, no. 6684, pp. 440-442, June 1998.

[7] A.-L. Barabási and R. Albert, "Emergence of Scaling in Random Networks," Science, vol. 286, no. 5439, pp. 509-512, October 1999.

[8] G. A. Pagani and M. Aiello, "From the Grid to the Smart Grid, Topologically," CoRR, vol. abs/1305.0458, 2013. [Online]. Available: http://arxiv.org/abs/1305.0458

[9] D. Deka, S. Vishwanath, and R. Baldick, "Analytical models for power networks:the case of the western us and ercot grids," IEEE Transactions on Smart Grid, vol. PP, no. 99, pp. 1-1, 2016.

[10] J. Hu, L. Sankar, and D. J. Mir, "Cluster-and-connect: An algorithmic approach to generating synthetic electric power network graphs," in 2015 53rd Annual Allerton Conference on Communication, Control, and Computing (Allerton), Sept 2015, pp. 223-230.

[11] E. Schweitzer, A. Scaglione, R. Thomas, and T. Overbye, "Analysis of the Coupling Between Power System Topology and Operating Condition for Synthetic Test Case Validation," in 2016 Grid of the Future Symposium. CIGRE US National Committee, 2016.

[12] Z. Wang and R. Thomas, "On bus type assignments in random topology power grid models," in System Sciences (HICSS), 2015 48th Hawaii International Conference on, Jan 2015, pp. 2671-2679.

[13] S. H. Elyas and Z. Wang, "Improved synthetic power grid modeling with correlated bus type assignments," IEEE Transactions on Power Systems, vol. PP, no. 99, pp. 1-1, 2016.

[14] M. Fiedler, "A property of eigenvectors of nonnegative symmetric matrices and its application to graph theory," Czechoslovak Mathematical Journal, vol. 25, no. 4, pp. 619-633, 1975.

[15] B. Mohar, "Laplace eigenvalues of graphs-a survey," Discrete Mathematics, vol. 109, no. 1-3, pp. $171-183$, 1992. [Online]. Available: http://www.sciencedirect.com/science/article/pii/0012365X9290288Q 
[16] S. Boyd, N. Parikh, E. Chu, B. Peleato, and J. Eckstein, "Distributed optimization and statistical learning via the alternating direction method of multipliers," Foundations and Trends $®$ in Machine Learning, vol. 3, no. 1, pp. 1-122, 2011.

[17] J.-P. Watson and D. L. Woodruff, "Progressive hedging innovations for a class of stochastic mixed-integer resource allocation problems," Computational Management Science, vol. 8, no. 4, pp. 355-370, 2011.

[18] R. Zimmerman, C. Murillo-Sánchez, and R. Thomas, "MATPOWER: Steady-State Operations, Planning, and Analysis Tools for Power Systems Research and Education," IEEE Transactions on Power Systems, vol. 26, no. 1, pp. 12-19, Feb 2011.

[19] R. Carlson and F. Fritsch, "Monotone piecewise bicubic interpolation," SIAM journal on numerical analysis, vol. 22, no. 2, pp. 386400, 1985. 